\title{
Author vs. Referee: A Case History for Middle Level Mathematicians
}

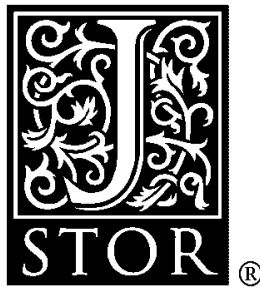

\author{
Robert C. Thompson
}

The American Mathematical Monthly, Vol. 90, No. 10. (Dec., 1983), pp. 661-668.

Stable URL:

http://links.jstor.org/sici?sici=0002-9890\%28198312\%2990\%3A10\%3C661\%3AAVRACH\%3E2.0.CO\%3B2-E

The American Mathematical Monthly is currently published by Mathematical Association of America.

Your use of the JSTOR archive indicates your acceptance of JSTOR's Terms and Conditions of Use, available at

http://www.jstor.org/about/terms.html. JSTOR's Terms and Conditions of Use provides, in part, that unless you have obtained prior permission, you may not download an entire issue of a journal or multiple copies of articles, and you may use content in the JSTOR archive only for your personal, non-commercial use.

Please contact the publisher regarding any further use of this work. Publisher contact information may be obtained at http://www.jstor.org/journals/maa.html.

Each copy of any part of a JSTOR transmission must contain the same copyright notice that appears on the screen or printed page of such transmission.

The JSTOR Archive is a trusted digital repository providing for long-term preservation and access to leading academic journals and scholarly literature from around the world. The Archive is supported by libraries, scholarly societies, publishers, and foundations. It is an initiative of JSTOR, a not-for-profit organization with a mission to help the scholarly community take advantage of advances in technology. For more information regarding JSTOR, please contact support@ jstor.org. 


\title{
AUTHOR VS. REFEREE: \\ A CASE HISTORY FOR MIDDLE LEVEL MATHEMATICIANS
}

\author{
ROBERT C. THOMPSON \\ Mathematics Department, University of California, Santa Barbara, CA 93106
}

This note evolved from a referee's rejection of a research paper that I wrote. The reasoning behind the rejection was perhaps unusual, and leads to a not altogether trivial question concerning the role of the referee in the professional development of a mathematician. The discussion will be more candid than is customary, and this may add spice to the article, since confession of failure, or even of sin, is always interesting.

The first section describes the mathematical problem that was investigated, and the second outlines what was proved concerning it and what was not. A research paper was written, and the motivation to publish it is given in the third section. The resulting referee's report is described in the fourth section, and in the fifth the somewhat psychological issues it leads to are examined. Those readers who submit papers to research journals may wish to reflect on these issues in the light of their own experience. The sixth section briefly discusses waiting times, and a few concluding remarks are in the seventh.

1. The Mathematical Question. Consider $n \times n$ matrices having integer entries. If $A$ is such a matrix, it is known that there exist unimodular matrices $U$ and $V$ of integers such that

$$
U A V=\left[\begin{array}{ccccccc}
a_{1} & & & & & \\
& a_{2} & & & 0 & \\
& & \cdot & & & \\
& 0 & & & \cdot & \\
& & & & & a_{n}
\end{array}\right]
$$

is a diagonal matrix with the diagonal elements forming a divisibility chain:

$$
a_{1}\left|a_{2}\right| \cdots \mid a_{n} .
$$

(The symbol $\mid$ signifies divides, with the convention that $0 \mid a$ means that $a=0$. To say that $U$ is unimodular means that $\operatorname{det} U= \pm 1$.) This is the diagonal form published by H. J. S. Smith in 1861 and now universally known as "the Smith canonical form." The diagonal entries $a_{1}, \ldots, a_{n}$ are called the invariant factors of $A$; they are unique to within a plus or minus sign. In the sequel $a_{1}, \ldots, a_{n-1}$ will always be taken nonnegative and $a_{n}$ will be chosen so that

$$
\operatorname{det} A=a_{1} a_{2} \cdots a_{n} \text {. }
$$

Let $B$ and $C$ also be $n \times n$ matrices of integers, with invariant factors

$$
\begin{array}{ll}
b_{1}|\cdots| b_{n} \text { for } B, & b_{1} \cdots b_{n}=\operatorname{det} B, \\
c_{1}|\cdots| c_{n} \text { for } C, & c_{1} \cdots c_{n}=\operatorname{det} C .
\end{array}
$$

The issue to be addressed is how invariant factors behave when matrices add. This question is significant because a great deal is known about the behavior of invariant factors under matrix multiplication, whereas essentially nothing is known about their behavior under addition. Write

Robert C. Thompson: I received my B.A. and M.A. degrees from the University of British Columbia and a Ph.D. from the California Institute of Technology. The thesis supervisors were Marvin Marcus (for the M.A.) and Olga Taussky Todd (for the Ph.D.) Most of my postdoctorate academic life has been at the University of California, Santa Barbara. My research program is concentrated in linear algebra, usually involving spaces over fields but sometimes over rings. I greatly enjoy teaching anything mathematical, and especially if there is an opportunity to present the pure mathematician's outlook to an applied audience. 
$C=A+B$; what does this imply about the $a_{i}, b_{i}, c_{i}$ ?

Since $a_{1}$ is the greatest common divisor of the elements of $A$ (similarly for $b_{1}^{\prime}$ and $B, c_{1}$ and $C$ ), an easy argument shows that any common factor of $a_{1}$ and $b_{1}$ is also a factor of every $a_{i}, b_{i}, c_{i}$ and of each element of $A, B, C$. It may therefore be cancelled. Thus generality will not be lost by taking $a_{1}, b_{1}, c_{1}$ pairwise relatively prime. This simplifying assumption will henceforth hold. To avoid trivial cases, take $a_{1}, b_{1}, c_{1}$ nonzero.

By Smith's theorem, there always exist unimodular matrices $U_{1}, U_{2}, V_{1}, V_{2}$ such that

$$
U_{1} A V_{1}=\left[\begin{array}{ccccc}
a_{1} & & & & \\
& . & & 0 & \\
& & . & & \\
& 0 & & \cdot & \\
& & & & a_{n}
\end{array}\right], \quad U_{2} B V_{2}=\left[\begin{array}{ccccc}
b_{1} & & & & \\
& . & & 0 & \\
& & . & & \\
& 0 & & \cdot & \\
& & & & b_{n}
\end{array}\right] \text {. }
$$

A consequence of this is the following equation in $2 n \times 2 n$ matrices:

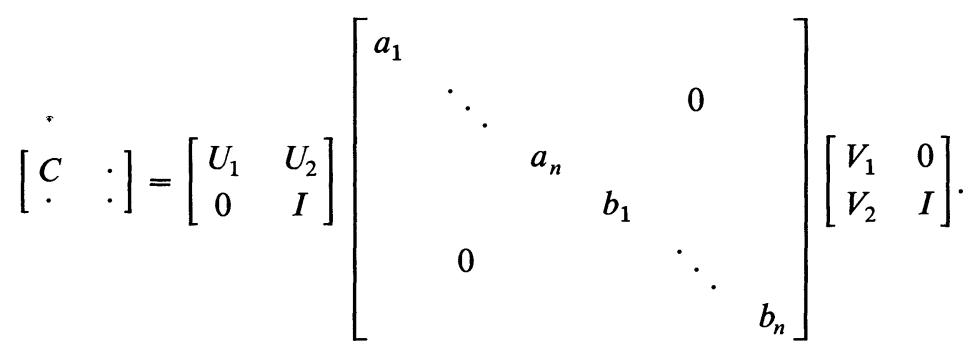

Since the first and last matrices on the right are unimodular, this equation reveals that $C$ is an $n \times n$ submatrix of a $2 n \times 2 n$ matrix having the same invariant factors as the diagonal matrix $D=\operatorname{diag}\left(a_{1}, \ldots, a_{n}, b_{1}, \ldots, b_{n}\right)$. Two facts will now be brought to bear:

(i) It is relatively easy, though not completely trivial, to prove that the invariant factors $d_{1}|\cdots| d_{2 n}$ of $D$ satisfy

$$
\left(a_{i}, b_{j}\right) \mid d_{i+j-1},
$$

where (,) signifies the greatest common divisor. The proof is in [6].

(ii) It is a somewhat standard fact, though not too well known, that the invariant factors $c_{1}|\cdots| c_{n}$ of $C$ and those $d_{1}|\cdots| d_{2 n}$ of the larger matrix containing $C$ satisfy

$$
d_{i} \mid c_{i} \text { for } 1 \leqslant i \leqslant n .
$$

These facts imply that

$$
\left(a_{i}, b_{j}\right) \mid c_{i+j-1}
$$

for all indices $i, j$ for which the subscripts lie in the range $1, n$.

The inequalities (= divisibility relations) visible in (1) are very much like a rather well-known family of inequalities for the eigenvalues of a sum or product of complex matrices. Example: If $A+B=C$, with $A, B, C$ Hermitian having eigenvalues $\alpha_{1} \geqslant \cdots \geqslant \alpha_{n}, \beta_{1} \geqslant \cdots \geqslant \beta_{n}, \gamma_{1} \geqslant$ $\cdots \geqslant \gamma_{n}$, respectively, then $\gamma_{i+j-1} \leqslant \alpha_{i}+\beta_{j}$. This lovely inequality was found by $\mathrm{H}$. Weyl in 1912 [9]. Extensions of it involving scatterings of terms are now known, the simplest being [5]

$$
\sum_{s=1}^{m} \gamma_{i_{s}+j_{s}-s} \leqslant \sum_{s=1}^{m} \alpha_{i_{s}}+\sum_{s=1}^{m} \beta_{j_{s}} \text { if } i_{1}<\cdots<i_{m}, j_{1}<\cdots<j_{m} .
$$

The indices on the terms here belong to a family of sequences occurring in the representation theory of the symmetric group and first found by D. E. Littlewood and A. R. Richardson in 1934 [2]. An immediate question is whether extensions of (1) along the lines of (2) are valid for the 
invariant factors of integer matrices. It turned out that most conjectured extensions of (1) were almost instantly seen to be wrong, and those that weren't could always be deduced from (1). Thus it appears that no further conditions like (2) have to be considered when studying the invariant factors of a sum of integer matrices.

But there is an obvious further condition of quite a different form. Modulo $a_{1}$, the matrix $A$ becomes zero, so that

$$
\operatorname{det} B \equiv \operatorname{det} C\left(\bmod a_{1}\right) \text {. }
$$

Observing now that everything so far said may be applied twice more by rewriting $A+B=C$ as $A+(-C)=-B$ and as $B+(-C)=-A$, we obtain the following theorem.

THEOREM 1. The invariant factors of a sum $A+B=C$ of integral matrices satisfy

$$
\left(a_{i}, b_{j}\right)\left|c_{i+j-1}, \quad\left(a_{i}, c_{j}\right)\right| b_{i+j-1}, \quad\left(b_{i}, c_{j}\right) \mid a_{i+j-1},
$$

$\operatorname{det} A \equiv(-1)^{n} \operatorname{det} B\left(\bmod c_{1}\right), \quad \operatorname{det} A \equiv \operatorname{det} C\left(\bmod b_{1}\right), \quad \operatorname{det} B \equiv \operatorname{det} C\left(\bmod a_{1}\right)$.

Question. Are the conditions of Theorem 1 sufficient?

That is, if integers $a_{1}|\cdots| a_{n}, b_{1}|\cdots| b_{n}, c_{1}|\cdots| c_{n}$ are given satisfying the conditions of Theorem 1, can integer matrices $A, B, C=A+B$ be found having the $a_{i}, b_{i}, c_{i}$ as their respective invariant factors? This was the mathematical problem that $I$ addressed in the research paper mentioned earlier.

2. Partial Answers. If $a_{i}, b_{i}, c_{i}$ are integers satisfying the conditions of Theorem 1 , can integer matrices $A+B=C$ be found with these integers as invariant factors? The matrices are to be $n \times n$; the following discussion treats each value of $n$ in turn. Recall the assumption made earlier that $a_{1}, b_{1}, c_{1}$ are pairwise relatively prime.

For $n=1$, the question has a negative answer: even though $a_{1}, b_{1}, c_{1}$ satisfy the conditions, they need not satisfy $a_{1}+b_{1}=c_{1}$. This discouraging result suggests that a reasonably clean theorem is unlikely.

For $n=2$, however, the answer is positive: If $a_{1}\left|a_{2}, b_{1}\right| b_{2}, c_{1} \mid c_{2}$ satisfy the conditions, it is possible to construct $2 \times 2$ matrices $A, B, C=A+B$ of integers having the $a_{i}, b_{i}, c_{i}$ as invariant factors. This encouraging result suggests that a reasonably clean theorem can probably be found.

The proof in the $2 \times 2$ case is not too hard, but not obvious either. Here are a few details. Since $a_{1}, b_{1}$ are relatively prime, there are integers $r, s$ such that $r a_{1}+s b_{1}=1$. Write

$$
A=a_{1}\left[\begin{array}{cc}
r c_{1} & b_{1} \\
b_{1} z_{21} & r c_{2}+b_{1} z_{22}
\end{array}\right], \quad B=b_{1}\left[\begin{array}{cc}
s c_{1} & -a_{1} \\
-a_{1} z_{21} & s c_{2}-a_{1} z_{22}
\end{array}\right], \quad C=\left[\begin{array}{cc}
c_{1} & 0 \\
0 & c_{2}
\end{array}\right],
$$

where $z_{21}$ and $z_{22}$ are integers to be chosen so that $A$ has $a_{1}, a_{2}$ as its invariant factors and $B$ has $b_{1}, b_{2}$. It is almost immediate that only the determinantal constraints $\operatorname{det} A=a_{1} a_{2}$ and $\operatorname{det} B=$ $b_{1} b_{2}$ have to be met. Using the conditions from Theorem 1 , this can be shown to be possible, after a not too long but not altogether straightforward analysis. There are nine conditions, but only three are actually needed, since these three imply the remaining six.

For $n=3$, the answer is again positive, but the proof is much, much longer. There are eighteen conditions, and all eighteen are needed, some repeatedly. The technique is similar to that used in the $2 \times 2$ case. One takes $C=\operatorname{diag}\left(c_{1}, c_{2}, c_{3}\right)$ to be diagonal,

$$
\begin{aligned}
& A=a_{1}\left[\begin{array}{ccc}
r c_{1} & b_{1} & b_{1} z_{13} \\
b_{1} z_{21} & r c_{2}+b_{1} z_{22} & b_{1} z_{23} \\
b_{1} z_{31} & b_{1} z_{32} & r c_{3}+b_{1} z_{33}
\end{array}\right], \\
& B=b_{1}\left[\begin{array}{ccc}
s c_{1} & -a_{1} & -a_{1} z_{13} \\
-a_{1} z_{21} & s c_{2}-a_{1} z_{22} & -a_{1} z_{23} \\
-a_{1} z_{31} & -a_{1} z_{32} & s c_{3}-a_{1} z_{33}
\end{array}\right],
\end{aligned}
$$


where $z_{13}, z_{21}, z_{22}, z_{23}, z_{31}, z_{32}, z_{33}$ are unknown integers to be chosen so that $A$ has $a_{1}, a_{2}, a_{3}$ as invariant factors ( $a_{1}$ is automatic) and $B$ has $b_{1}, b_{2}, b_{3}$. The task is a difficult diophantine problem, and it is not obvious how to proceed. Nevertheless, I found a way of proceeding, in a proof that runs for about eleven typed pages. I believe this proof to be a reasonably substantial achievement.

I made some half-hearted attempts to settle the case $n=4$, and I failed. I was, frankly, tired of the problem after the arduous work needed to settle the $3 \times 3$ case. So, sufficiency of the conditions of Theorem 1 is not established for $n \geqslant 4$.

A comment is in order concerning the cases $n=2$ and $n=3$. The proofs just described failed to reveal any conceptual framework within which to set the problem, and so reveal few genuine clues showing how to proceed to higher dimensions. The arguments are clever, but perhaps only ad hoc. The lack of a conceptual framework is very distressing. What is the structural foundation? None is known to me.

3. The Impetus Toward Publication. At this point, I came under review for a salary increase at my university, and to justify the increase it was of course necessary to exhibit a lot of new papers. (Unfortunately, quality is hard to establish, thus quantity counts in these matters.) I got busy and wrote up a dozen papers, selecting the dozen items easiest to write up from a store of unpublished results. The invariant factor problem was one of the dozen, and was written up as a manuscript entitled Sums of integral matrices. Because of the great difficulty I had experienced constructing the $3 \times 3$ sufficiency proof, I was absolutely confident that the paper would be accepted without hesitation. It was submitted to the Rocky Mountain Journal of Mathematics.

4. The Referee's Report. The referee's report was short, and its contents were quite unexpected. Here it is (with a journal reference deleted):

I suggest not publishing the enclosed paper of R. C. Thompson in the Rocky Mountain Journal of Mathematics.

The problem he is looking at is: To what extent do the invariant factors of integral matrices $A$ and $B$ determine those of $A+B$ ? The problem is moderately interesting. But he hasn't solved it: He has only done the 2 by 2 and 3 by 3 cases. The computation that decides these rather special cases is a real tour-de-force, but doesn't seem to uncover any interesting ideas.

Thompson has done some very interesting work on integral matrices (e.g., his paper on interlacing inequalities for invariant factors). Basically, I feel someone as good as him should not try to publish such a partial, unilluminating result.

Evidently the referee read the paper in detail, and understood it thoroughly. I interpret his report in this way: "Thompson is capable of solving the full problem, and he should get busy and do so!"

The next section, which should be regarded as the heart of this note, will discuss the referee's report from various points of view. Everything so far has merely been to set the background for the discussion. How do you respond to the issues about to be raised?

\section{Discussion.}

Disclaimer. Both the referee and I may well have missed the point: there may be a more or less easy solution for the invariant factor problem. The reader is challenged to find one. If one is found, the following discussion may lose its punch, but this note will still have merit since it provided the stimulus for the reader's creative effort.

To continue, however, assume that no easy solution is possible, so that the results in hand are a nontrivial partial solution to a difficult problem.

Criteria for publishability: are there any generally accepted ones? They would appear to be enough depth or breadth, plus a write-up possessing sufficient clarity and polish. Originality is a key item, and great originality will offset a presentation lacking clarity or polish.

Responsibilities of an author: what are they? They are to ensure that sufficient originality is 
present, that the manuscript is technically accurate, that it is carefully prepared, and that incomplete partial results are not offered when more can be achieved with a not unreasonable additional effort.

A side comment: Every mathematician serving in an editorial capacity soon learns that many prospective authors fail to discharge these responsibilities adequately; some fail grossly. Premature submission of partial results is a common sin, and inadequate attention to the technical aspects is another. (Are the proofs correct? Has the manuscript been properly proofread? Is the grammar sound?) Unreadable manuscripts are all too common. Some writers seem to feel that referees are servants, to take care of details that were overlooked. No judgment could be more mistaken.

Responsibilities of a referee: what are they? They are to certify that the generally accepted standards are met, to suggest improvements, and to act as a stimulus to cajole an author into a further creative effort. A referee should be able to recognize the worth of mathematical styles and attitudes differing from his own. No referee, however, has to be an author's servant, correcting shortcomings caused by carelessness, immediate rejection being the proper action in such cases. If, however, the referee is overworked, it is sound procedure to rely on an author's reputation (if any) for quality and accuracy. This does not mean that an established mathematician should expect publication of his second class results. And every referee should be willing to give a helping hand to a novice writer.

(The harsh sentences in the two paragraphs above are caused by this author's experience as a journal editor.)

Consider now the referee's reports in the light of the headings above. Because my manuscript was prepared with extreme care, the quality of the presentation and the technical aspects were probably adequate. I shall assume this to be the case since the referee made no comment to suggest otherwise. I now pose, and partially answer, five somewhat psychological questions.

Question 1. Should a nontrivial partial solution of a difficult problem be published?

Many mathematicians would say "yes," but some probably would say "no." What is your opinion? The referee seemed to feel that the answer is "no," but perhaps he was meeting his duty of cajoling the author into a greater creative effort. In general, I feel that a partial solution should be published if lack of publication would risk loss of a nontrivial piece of work. The whole purpose of our professional existence is to get problems solved, and if a partial solution will contribute significantly to an ultimate complete solution that may happen many years later, then the permanent record created by publication is justified.

(Rule out publishing simply to create a long publication list: that's not unknown, and probably unprofessional, even though sometimes dictated by university promotion policies. I have at times been guilty of the sin. Are you also guilty?)

Question 2. Should the lack of a structural basis affect the merit of the results so far obtained?

The referee appeared to feel that this was the case. But mathematics is full of examples of difficult questions for which no structural framework has been found. Does this lack render the questions less interesting? Many mathematicians, those who are problem solvers rather than theory developers, would say that the lack of a structural setting adds to the appeal. The absolutely pure battle between mathematician and nature, without the corrupting influence of a lot of distracting structure, is surely the highest form of intellectual activity. So the referee appears to be on shaky ground with this aspect of hís analysis. It is true that the proper conceptual framework might make the problem solvable, or at least accessible (as is the case for the question of how invariant factors behave when matrices multiply, a question leading to Young tableaux and Littlewood-Richardson sequences), but it might also reveal it to be difficult. What is your opinion on this issue?

Question 3. Was the attempted publication premature since only low dimensional cases were solved? 
The referee probably felt that it was, and it might have been. However, I was willing to publish a partial result and abandon the invariant factor problem because I was attracted to some other equally difficult problems-see below.

Do you sometimes abandon a problem with only a partial solution because something else becomes more interesting, or because you feel unable to achieve more? If so, what do you do with your partial result?

(One response, which I quite deliberately adopted in another case when three years of intermittent effort yielded only a partial result, is to submit a manuscript containing conjectures and those facts that can be proved, hoping not for an acceptance but for a referee's report containing a significant idea. It is permissible to use the referee's talents, provided his contributions are acknowledged. If he cannot supply an idea, a case for publication may already be established. Can you guess how this gambit worked for me? Answer below.*)

Question 4. A referee might suggest that the author solve a problem in full, but the author has other problems that he wishes to attack, problems that he finds more attractive. Which course of action should the author follow?

This question could be rephrased as: "How much influence should a referee have in the professional development of a mathematician?" In the present instance, the dilemma is partly attributable to my formulation of the following conjecture, a conjecture that is probably deep and certainly very structural.

\section{CONJECTURE. If $A$ and $B$ are Hermitian matrices, there will always exist unitary matrices $U$ and $V$ so that}

$$
e^{i A} e^{i B}=e^{i\left(U A U^{-1}+V B V^{-1}\right)},
$$

where, of course, $i=\sqrt{-1}$.

The conjecture belongs to the interface between Lie groups and Lie algebras and can be formulated in Lie-theoretic terms. I have many partial results concerning it. The Campbell-BakerHausdorff formula $e^{x} e^{y}=e^{z}$ (where $z$ is a formal series in commutators of $x$ and $y$ ) of course plays a role. The conjecture can also be formulated for non-Hermitian matrices $A, B$, but then it can only hold for $A, B$ in a neighborhood of 0 . I have not published my partial results on this class of questions because I hope (perhaps unrealistically!) to produce a complete solution. But one thing is clear: if I work more on conjecture (3), I will have to commit all my resources to it, and cannot work on the invariant factor problem. It's one or the other, not both, since I am (unfortunately) only an average mathematican, far, far below the leaders. Should I be influenced by the referee's wishes that the invariant factor problem be solved in full?

(It is possible that a reader of this article who is expert in Lie theory may resolve conjecture (3). If that happens, I shall return to the invariant factor problem, unless it too is settled by a reader!)

Question 5. Will an author lose prestige in the eyes of a referee if a rejected paper is submitted to another journal without change? If so, should the author be influenced by this fact?

That's a tough issue, the possible loss of prestige being the only reason that I have not resubmitted Sums of integral matrices. In fact, I have an invitation from another journal to publish it there, in its present form, and if this were not the case, I could publish it in the journal I edit, Linear and Multilinear Algebra. But I have done nothing. What would you do in this situation?

*Answer. The paper, [8], contained a conjecture having every appearance of being solvable in a few lines. The referee took 14 months to prepare a very accurate report, recommending acceptance, but not furnishing the hoped for idea. The journal's editor, noting the referee's observation that the conjecture ought to have a short solution, chose to overrule the recommendation and rejected the paper. It remains unpublished and the conjecture unsolved. Clearly, the editor may be a major factor in the author-referee configuration. 
The note you are presently reading-Author vs. referee-is substantially based on a lecture that I gave at the matrix conference run by S. Pierce at the University of Toronto, August, 1982. Some members of the audience felt that the paper should immediately be resubmitted, but others could understand my reluctance to do so.

There may be other questions that arise naturally. Do any occur to you? Here is one: The world of mathematics is populated mainly by average mathematicians, since the very best of us are so few. Journals obviously cater to the very best. To what extent should they cater to the average? One may argue that civilized society needs mathematicians, and that the middle level mathematician undoubtedly is a better contributor to society if journals exist that will publish his middle level theorems. What is your opinion?

6. Waiting Times. How long should an author wait before expecting to receive a referee's report? Many authors expect a report within a few months, and some expect one sooner. A clearly written paper almost always gets a report more quickly than a badly written one, and to this extent an author can do a lot to help his own case. My experience with papers that I have submitted to journals is: minimum waiting time until an editorial decision is reached - $a$ few days, maximum-four years, typically - a few months. The four year wait led to an acceptance, probably attributable to the referee's sense of guilt. To this extent, it may pay to hope for a referee who is not speedy.

A fair rule of thumb seems to be. A referee is entitled to six months in which to prepare his report, but not longer. This means: an author should not start to complain until six months have elapsed. The six month value is somewhat arbitrarily chosen. What do you think the correct figure should be?

Some mathematicians are very conscientious about meeting their obligations as referees. Others are extremely remiss. It really seems unfair that the latter cannot be somehow penalized. Is there an imaginative journal editor somewhere who can invent the appropriate punishment?

How long did the referee of Sums of integral matrices take? About six weeks, an excellent performance in view of the difficult $3 \times 3$ proof.

\section{Some Comments.}

(i) The invariant factor theorems attributed to me in the referee's report were also obtained by E. Marquis de Sa [3], and certain parts of them can be deduced from facts in Bourbaki. The referee should have known that! (Perhaps he did.)

(ii) The question of the similarity invariant factors of a sum of matrices with entries in a field has been studied in [1] with further results in the doctoral thesis of E. Marquis de Sa.

(iii) Did I get my salary increase? I did, and fortunately it was granted before the rejection was received, making it unnecessary to jeopardize the increase by having to remove an item from my publication list. (Are you as lucky?)

(iv) Of the dozen papers mentioned above, how many were accepted? Obviously not all, and the exact number of acceptances will be left for the reader to guess. It is true, though, that some of the accepted papers were weaker than the rejected one discussed in this article. This seems to imply that not all referees adhere to the same standards. Should an author resubmit a rejected paper, hoping for an "easier" referee? The tactic is surely not unknown. Does it conform to established standards of professional conduct? Probably not. A much better ploy is to make the paper stronger, revising as necessary, before resubmitting. However, an author is sometimes (not always!) a better judge of the mathematical situation than the referee: If the contents of a rejected paper form a step in a well conceived campaign toward a substantial objective, resubmission without change may be justified.

The lack of a universal standard for evaluating mathematical papers was visible in the opinions expressed by the four referees of the article before you, Author vs. referee. The report given me by the MoNTHLY's editor suggests that of the positions taken by the four about Sums of integral matrices, one supported me, one supported the referee, one could see both sides of the issue, and 
the position of the fourth was not disclosed. So much for unanimity! The four did not agree on the merit of Author vs. referee either.

Sometimes a referee's judgement is later seen to be wrong. Now and then I am sent manuscripts to appraise, and I do reject some. Two that I rejected were later published without change in other journals, and in retrospect I think both authors took the correct path. Mistakes do occur!

(v) It is to be emphasized that I bear no grudge against the referee for rejecting my paper. After many years as a professional mathematician, I have learned to accept such twists of fate stoically. A major point is that the rejection has created uncertainty in my mind: should I continue with the invariant factor problem, or abandon it for pastures that I presently perceive to be more attractive? (Can you be similarly unmoved when one of your papers is rejected? The correct, professional, response following a rejection is to examine objectively the reasons behind it.)

(vi) No statistics seem to be available on journal rejection rates. An informed guess is that most journals reject roughly $40 \%$ of submitted manuscripts, and some (including the Monthly) much more. Do you think too many manuscripts are rejected? Or too few?

(vii) I believe this article to be in good taste, even though rather candid, addressing an annoying fact of life with which most middle level mathematicians must contend. Do you agree? Or should it have been rejected? Readers may send comments to me.

Summary. The interface between author and referee is an uneasy one.

\section{References}

1. G. N. de Oliveira, E. Marques de Sa, and J. A. Dias da Silva, On the eigenvalues of $A+X B X^{-1}$, Linear and Multilinear Algebra, 5 (1977) 119-128.

2. D. E. Littlewood and A. R. Richardson, Group characters and algebra, Philos. Trans. Roy. Soc. London Ser. A, 233 (1934) 99-141.

3. E. Marques de Sa, Imbedding conditions for $\lambda$ matrices, Linear Algebra Appl., 24 (1979) 33-50.

4. H. J. S. Smith, On systems of linear indeterminate equations and congruences, Philos. Trans. Roy. Soc. London Ser. A, 151 (1861) 293-326; Collected Works I, 367-409.

5. R. C. Thompson (with L. J. Freede), On the eigenvalues of a sum of Hermitian matrices, Linear Algebra Appl., 4 (1971) 369-376.

6. R. C. Thompson, The Smith invariants of a matrix sum, Proc. Amer. Math. Soc., 78 (1980) 162-165.

7. R. C. Thompson, Sums of integral matrices, manuscript.

8. R. C. Thompson, $p$-adic matrix valued inequalities, manuscript.

9. H. Weyl, Das asymptotische Verteilungsgesetz der Eigenwerte linearer partieller Differentialgleichungen, Math. Ann., 71 (1912) 441-479.

\section{MISCELLANEA}

115.

To see ourselves as others see us?

"If you try to understand fibre bundles by reading mathematics, if you are a physicist, you would probably not succeed, because modern mathematics is extremely difficult to read, and I believe there exist only two kinds of modern mathematics books; one which you cannot read beyond the first page and one which you cannot read beyond the first sentence."

-C. N. Yang, Lectures on Frontiers in Physics, Seoul, Korea, 1980. 